\title{
Assembly-Controlled Biocompatible Interface on a Microchip: Strategy to Highly Efficient Proteolysis
}

\author{
Yun Liu, ${ }^{[a, d]}$ Wei Zhong, ${ }^{[b, d]}$ Sheng Meng, ${ }^{[b]}$ Jilie Kong, ${ }^{[a]}$ Haojie Lu, ${ }^{[a]}$ Pengyuan Yang, ${ }^{[a]}$ \\ Hubert H. Girault, ${ }^{[\mathrm{c}]}$ and Baohong Liu*a]
}

\begin{abstract}
A biocompatible interface was constructed on a microchip by using the layer-by-layer (LBL) assembly of charged polysaccharides incorporating proteases for highly efficient proteolysis. The controlled assembly of natural polyelectrolytes and the enzyme-adsorption step were monitored by using a quartz-crystal microbalance and atomic force microscopy (AFM). Such a multilayer-assembled membrane provides a biocompatible interconnected network with high enzyme-loading capacity. The maxi-
\end{abstract}

mum digestion rate of the adsorbed trypsin in a microchannel was significantly accelerated to $1600 \mathrm{~mm} \mathrm{~min}^{-1} \mu \mathrm{g}^{-1}$, compared with the tryptic digestion in solution. Based on the Langmuir isotherm model, the thermodynamic constant of adsorption $K$ was calculated to be $1.6 \times 10^{5} \mathrm{M}^{-1}$ and the maximum adsorption loading $\Gamma_{\max }$

Keywords: interfaces • layer-by-layer assembly $\cdot$ microchip reactors . proteomics $\cdot$ trypsin was $3.6 \times 10^{-6} \mathrm{molm}^{-2}, 30$ times more than a monolayer of trypsin on the native surface. The tunable interface containing trypsin was employed to construct a microchip reactor for digestion of femtomoles of proteins and the produced peptides were analyzed by MALDI-TOF mass spectroscopy. The efficient on-chip proteolysis was obtained within a few seconds, and the identification of biological samples was feasible.

\section{Introduction}

The construction of a functionalized surface on various substrates is critical for biological analyses, including clinical and cellular assays, and enzymatic reaction. ${ }^{[1-3]}$ Appropriate functional groups that have a high affinity for biomolecules offer alternative opportunities for high-throughput and sensitive identification of specific binding sites of biological interaction events. The main challenge is to find a general ap-

[a] Y. Liu, Prof. J. Kong, Dr. H. Lu, Prof. P. Yang, Prof. B. Liu Department of Chemistry

Institute of Biomedical Sciences, Fudan University

Shanghai 200433 (P. R. China)

Fax: (+86) 21-6564-2405

E-mail: bhliu@fudan.edu.cn

[b] Dr. W. Zhong, S. Meng

Department of Macromolecular Science, Fudan University

Shanghai 200433 (P. R. China)

[c] Prof. H. H. Girault

Laboratoire d'Electrochimie Physique et Analytique

Ecole Polytechnique Federale de Lausanne 1015 Lausanne (Switzerland)

[d] Y. Liu, Dr. W. Zhong

These authors contributed equally to this research. proach to engineer surfaces with targeted specific properties, such as tunable reactivity, biocompatibility, or wettability. ${ }^{[4]}$

Microfluidic devices are becoming powerful tools for performing chemical or biological assays, due to the increased speed and reliability at reduced sample consumption. The continuing progress in microchip-based bioanalysis will depend on the development of novel surface-modification technologies in a simple and reliable fashion. The chemical patterning of a biocompatible interface within microfluidic channels must be efficient to immobilize domains of antibodies, enzymes, and other important biologically active compounds for a highly sensitive detection. In particular, as protein analysis continues to push the limits, the availability of new strategies will become more critical. Proteolysis is the key step for positive protein sequencing in proteomics research integrated with MALDI-TOF MS. The conventional techniques of in-solution digestion of proteins offer limited sensitivity and are time-consuming procedures, affecting severely the determination of comprehensive proteomic profiles. $^{[5]}$

One of the promising solutions is the incorporation by patterning processes of enzymes, such as proteases, within a microchannel to form a microbioreactor to carry out highly efficient and low-level protein digestion. Relative to free en- 
zymes in bulk solution, the enzymes immobilized in a microchannel are more stable and resistant to environmental changes, and provide molecular-level interactions with flowing substrates. The heterogeneity of the immobilized enzyme systems allows multiple reuses of the enzymes, continuous operation of enzymatic processes, and a greater variety of bioreactor designs. Several approaches, such as physical adsorption, sol-gel encapsulation, and covalent linking, have been explored to immobilize enzymes onto the walls of microfluidic channels to design reactors for proteolysis. ${ }^{[6,7]}$ Another alternative and facile option is the multilayer-assembly technique established by the layer-by-layer deposition in a controlled way. ${ }^{[8]}$ The layer-assembled microstructures with tailored composition and architecture can be used to incorporate functional biomolecules. ${ }^{[9]}$ Biomolecules embedded into the polyelectrolyte mutilayers are close to their native form, so that the biological films have many potential applications, such as biosensors and immunoassays.

Here, a biocompatible interface was constructed on a poly(ethylene terephthalate) (PET) microfluidic chip providing a miniaturizing and viable platform for the immobilization of biomolecules. The PET microchannel is functionalized by the alternating deposition of two naturally occurring polysaccharides, the cationic chitosan (CS) and anionic hyaluronic acid (HA). In view of their biocompatibility, nontoxicity, and hydrophilicity, the CS/HA-assembled networks offer the unique characteristic of affinity to enzymes or proteins. So far, they have been usually used for tissue engineering or drug delivery. ${ }^{[10]}$ We have proposed a simple method with this self-assembly for imbedding enzymes on microchips for protein identification. ${ }^{[7 c]}$ The present investigation is focused on the characterization of enzyme-loading multilayer assemblies within a microchannel for the sensitive proteolysis of standard proteins at lower detection limits and also of real biological samples. The constructed microreactor provides a large surface-area-to-volume ratio and a confined microenvironment, resulting in an increased reaction rate. Within a few seconds, the peptides derived from digestion of proteins are detected with high sequence coverage. Biological samples, such as casein extracted from bovine milk and attenuated hepatitis A virus vaccine, were identified by using this proposed microchip reactor coupled with MALDI-TOF MS. This strategy could be applied potentially as an on-chip microreactor for challenging other techniques for low-abundance proteome analysis, as well as the rapid analysis of real-world samples.

\section{Results and Discussion}

The PET substrate, a commonly used polymer material, has been employed to fabricate microfluidic chips by laser ablation for practical proteomics purposes interfaced to mass spectroscopy, electrochemistry, and fluorescence detection. ${ }^{[1]}$ However, the PET surface properties of hydrophobicity and poor biocompatibility are serious obstacles for bioanalysis. Thus, the chemical modification of PET microchannel surfaces is essential. In the present study, a simple modification protocol is proposed for an enzyme multilayer adsorption on microchips to obtain a microreactor for online proteolysis. Scheme 1 describes the process of enzyme immobilization and protein identification in the multilayerassembled microchip.

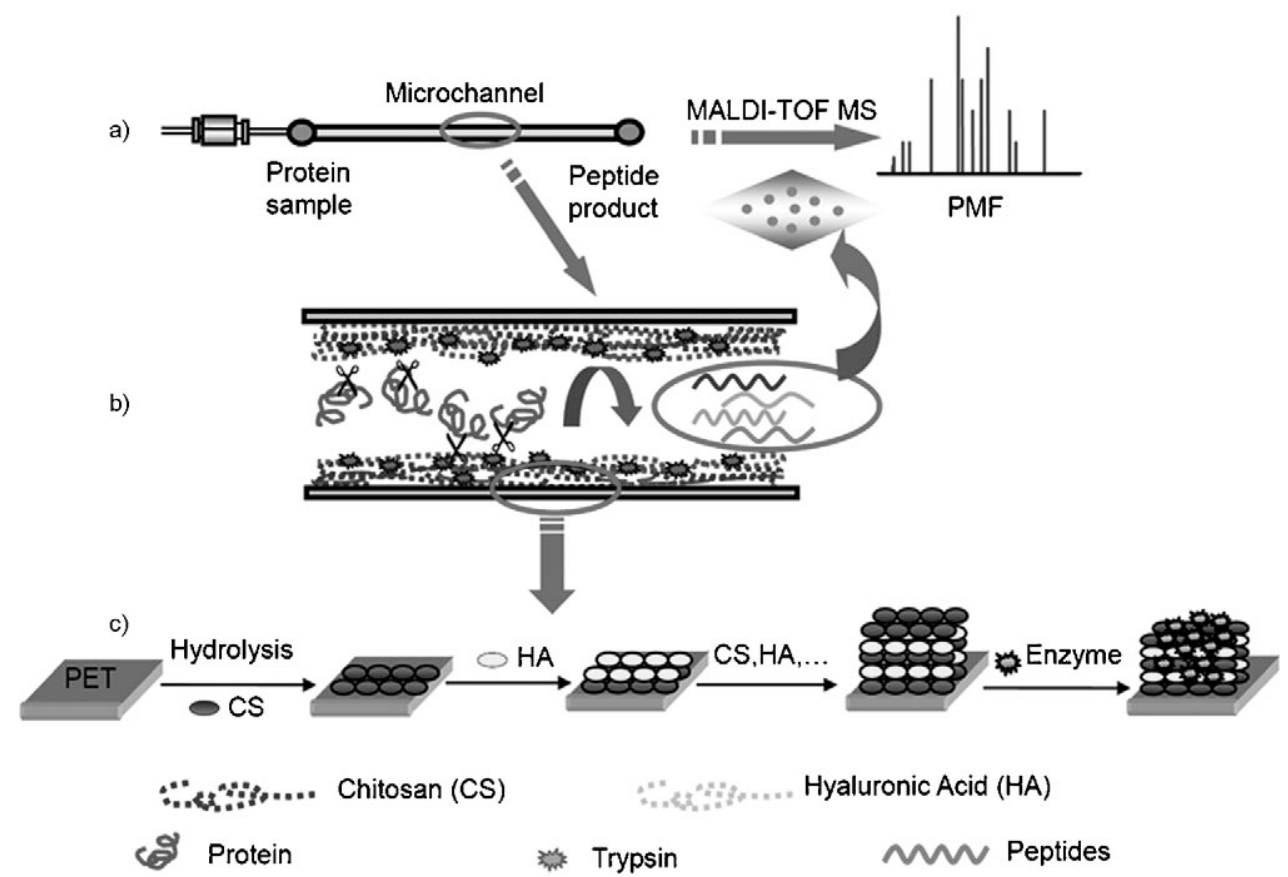

Scheme 1. a) On-chip protocol for protein digestion and identification coupled with MALDI-TOF MS. b) Amplified multilayer-assembled enzyme microchip reactor within microchannel. c) Depiction of layer-by-layer process for enzyme immobilization. 
Several bilayers of CS and HA were prepared to produce a stable supramolecular thin film by electrostatic interaction. Atomic force microscopy (AFM) images present topographical features of the multilayer-assembled PET surface containing the enzyme. As shown in Figure 1a, the surface of native PET is rather flat and smooth with few defects, and the RMS roughness is about $87 \AA$. In comparison, after deposition of CS/HA multilayer films on the PET surface, the image reveals uniform protrusive structures with a roughness of $318 \AA$ (Figure $1 \mathrm{~b}$ ), among which pores with a size of several tens to hundreds of nanometers are quite evenly distributed on the surface. Such a well-interconnected porous network is suitable for incorporating enzymes whilst maintaining easy pathways for protein access and peptide diffusion. ${ }^{[12]}$ Trypsin, used here as the protease, has an approximate crystallographic size of $4.0-5.0 \mathrm{~nm} \cdot{ }^{[13]}$ Figure $1 \mathrm{c}$ displays the surface morphology of trypsin-loaded CS/HA multilayers, with pores of a few nanometers and smoother than that exhibited in Figure 1b. This illustrates that the trypsin was embedded into the microstructured network of CS/HA multilayers, and adsorbed onto the CS/HA-modified PET surface.

The adsorbed mass of CS/HA and trypsin was monitored by using the quartz-crystal microbalance (QCM) frequency shift according to the Sauerbrey relation. Experimental data obtained by using a gold electrode show that the gradual $\mathrm{CS} / \mathrm{HA}$ multilayer growth is a linear function of the number of layers (Figure 2, inset). Layer-by-layer (LBL) deposition driven by electrostatic forces can easily take place on a model surface, which will provide the enzyme with a suitable microenvironment. The first layers (CS1/HA2/CS3/HA4/ CS5) offer a supporting precursor film for better adsorption of the enzymes on the surface. As shown in Figure 2, a continuous decrease of the QCM frequency shift as the number of enzyme layers increases confirms the successive adsorption of enzymes on the functional scaffold by means of CS/ HA multilayer buildup. After deposition of the second HA/ CS-trypsin layer, the frequency decrease reaches a plateau. We speculate that the first enzyme layer is within an incompact microenvironment, and subsequently, the enzymes adsorb mainly onto the surface of the multilayer and do not

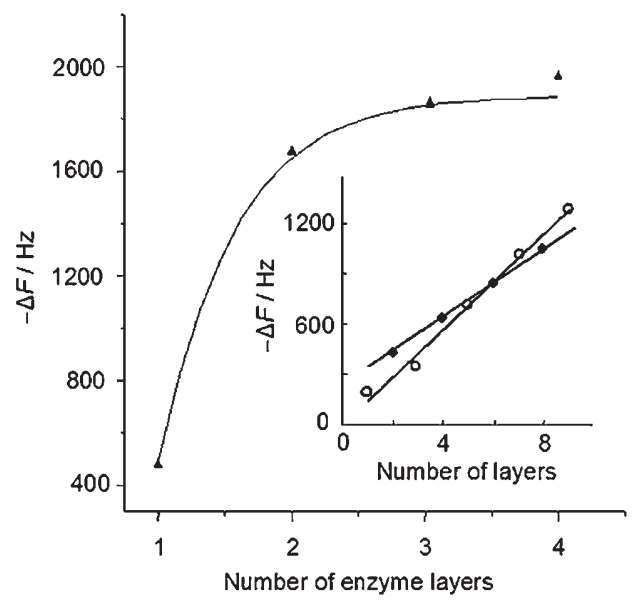

Figure 2. QCM frequency change as a function of the number of enzyme layers. One layer corresponds to the assembly CS1/HA2/CS3/HA4/CS5/ trypsin, two to four after addition of HA/CS-trypsin layers. Inset: QCM frequency change of a bare multilayer assembly as a function of the number of polyelectrolyte layers. CS (०), HA $(\bullet)$.

penetrate the multilayer fully as the number of layers increases. Nevertheless, the amount of adsorbed enzyme increases as the deposition of multilayers (HA/CS-trypsin) continues. ${ }^{[7]}$ Four trypsin layers correspond to an enzyme loading of about $107 \mathrm{mg} \mathrm{m}^{-2}$, higher than with other polyelectrolytes. ${ }^{[14]}$

Adsorption and transport processes were modeled mathematically to gain a better understanding of the behavior of enzyme adsorption onto the multilayer-assembled substrates. The Langmuir isotherm model has a wide application for the adsorption of proteins on substrates, which is useful in practice. ${ }^{[15]}$ According to the Langmuir adsorption isotherm equation:

$\Gamma_{\text {eq }} / \Gamma_{\max }=K C^{\mathrm{o}} /\left(1+K C^{\mathrm{o}}\right)$

in which $\Gamma_{\mathrm{eq}}$ is the surface concentration at equilibrium, $\Gamma_{\max }$ is the initial concentration of the active sites, $K$ is the thermodynamic constant of adsorption, and $C^{\circ}$ is the initial concentration of the solution. The experimental isotherm of

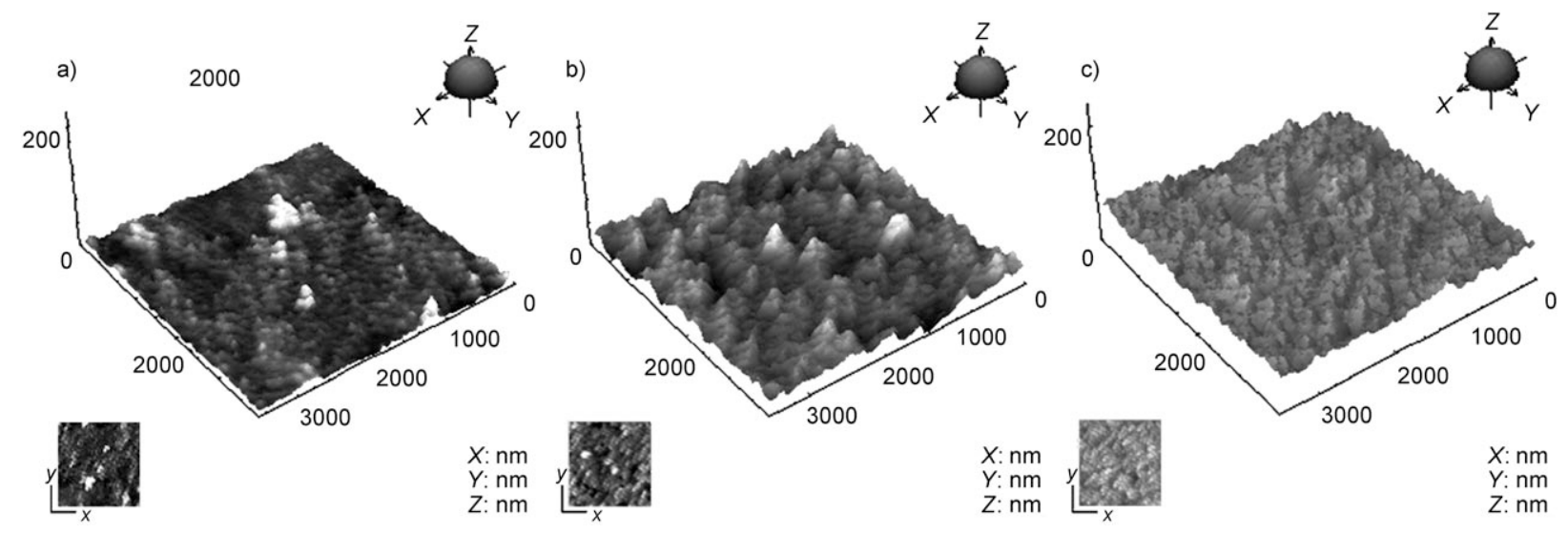

Figure 1. AFM images (tapping mode) of the PET surface (a) untreated, (b) following deposition of CS/HA multilayers with the chitosan as the outermost layer, and (c) trypsin immobilized on the LBL assembly-modified PET. 
trypsin adsorption is shown in Figure 3a, and the linearized isotherm is the inset line. By plotting $C^{\mathrm{o}} / \Gamma_{\mathrm{eq}}$ versus $C^{\mathrm{o}}, \Gamma_{\max }$ and $K$ are provided as the respective reciprocals of the slope and the intercept, giving the fitted values $\Gamma_{\max }=3.6 \times$ $10^{-6} \mathrm{~mol} \mathrm{~m}^{-2}$ and $K=1.6 \times 10^{5} \mathrm{M}^{-1}$. Furthermore, we suppose


Figure 3. a) Isotherm of trypsin adsorption on the multilayer-assembled PET obtained from the QCM experimental results. Inset line: linearization of the adsorption isotherm. b) The maximum tryptic rate as a function of the number of HA/CS-trypsin layers.

a compact monolayer of trypsin molecules on the native surface. The maximum adsorption $\Gamma_{\max }{ }^{\circ}$ can be calculated by taking into account the cross-section area of a trypsin molecule (a trypsin molecule is globular ${ }^{[16]}$ with a diameter of about $\left.4-5 \mathrm{~nm}^{[13]}\right)$, resulting in $1.0 \times 10^{-7} \mathrm{~mol} \mathrm{~m}^{-2}$. The fitted value $\left(\Gamma_{\max }=3.6 \times 10^{-6} \mathrm{~mol} \mathrm{~m}^{-2}\right)$ obtained from the multilayer-assembled system is 30 times more than for the monolayer of trypsin on the unmodified surface. These results demonstrate that the HA/CS network on the wall of the microchannel provides a porous medium for enzyme loading.

This interface provides a well-defined scaffold for enzyme immobilization, in which the bioactivity is assumed not to be compromised. Accordingly, the adsorbed trypsin activity is characterized by the values of the Michaelis constant and maximum velocity, which are usually derived from the Michaelis-Menten equation. As shown in Figure 3b, enzyme activity increases steeply from one layer to three layers. However, after the deposition of a third HA/CS-trypsin layer, the activity reaches a plateau, because the diffusion and permeation of substrate into the membrane is more lim- ited as the number of layers increases. ${ }^{[14]}$ Four enzyme-loading layers showed an activity of $7.85 \mathrm{~mm} \mathrm{~s}^{-1}$, which is of a similar order of magnitude to those reported for a trypsinencapsulated reactor on porous polymer monoliths. ${ }^{[6]}$ The value of maximum velocity $\left(V_{\max }\right)$ per unit trypsin was about $1600 \mathrm{~mm} \mathrm{~min}^{-1} \mu \mathrm{g}^{-1}$, thousands of times faster than in bulk solution. Both a high surface-to-volume ratio and microstructured confinement within the microchannel induce order-of-magnitude enhancement of catalytic efficiency. In addition, the significantly increased velocity in the functionalized microchannel may also be attributed to the fact that the well-defined interface assembled by CS/HA could provide not only a large surface area for enzyme immobilization, but also a biocompatible microenvironment for preserving the bioactivity and reducing the autolysis, which is promising for sensitive analysis.

The CS/HA-assembled microchip serving as an on-chip enzyme reactor coupled to MALDI-TOF MS could be a facile strategy for efficient digestion. Proteins, such as bovine serum albumin (BSA), myoglobin, cytochrome c (Cyt-c), casein extracted from bovine milk, and attenuated hepatitis A virus vaccine, are exemplified for the feasibility of this approach. The tryptic efficiencies were evaluated on the basis of the protein identification results with the detected peptide mass fingerprinting (PMF) and signal-to-noise ratios.

Figure 4a displays the PMF spectra of tryptic digests of $200 \mathrm{ng} \mu \mathrm{L}^{-1} \mathrm{BSA}$ with the as-prepared microreactor, and in solution as a control. At a flow rate of $120 \mu \mathrm{Lh}^{-1}$, a total of 28 tryptic peptides from BSA were assigned and correspond to an amino-acid sequence coverage of BSA as high as $43 \%$. Relative to proteolysis in solution (Figure $4 \mathrm{~b}$ ), both the signal-to-noise ratio and sequence coverage are clearly enhanced, and the reaction time is reduced to a few seconds. The results show that trypsin entrapped in the PET microchannels can act as a good biocatalyst and can achieve more rapid reaction.

This strategy has potential in the identification of protein at low abundance. As the substrate concentration is reduced, the kinetics of the reaction are reduced to a point at which a longer digestion time is required. To decrease the digestion time, the concentration of enzyme can be increased. However, the presence of a high enzyme-to-sample ratio often produces a severe enzyme autolysis, which can occlude the signals of the sample peptides in the spectrum. ${ }^{[17]}$ There has been speculation that the enzymes immobilized into a microfluidic channel are more stable than their soluble forms because of the suppression of autolysis by the matrix. Naturally expressed proteins at low abundance play key roles in cells and tissues. In the interest of achieving highly specific activity, while significantly minimizing reaction time with the microchip reactor, the digestion efficiency of proteins at a low level will be enhanced. The mass spectrum of digests of $1 \mathrm{ng} \mu \mathrm{L}^{-1}$ myoglobin is shown in Figure $5 \mathrm{a}$. The amino-acid sequences of $34-40 \%$ and protein scores of 119-145 are detected, and no enzyme autodigestion signals are observed in the spectra. The lower tryptic 


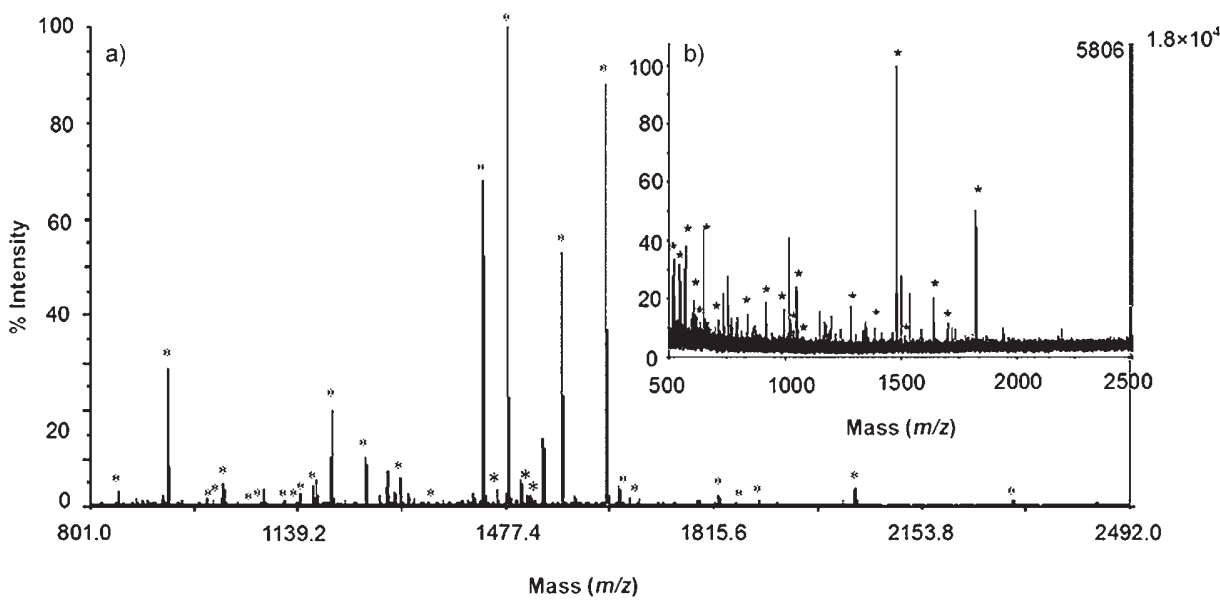

Figure 4. a) MALDI-TOF mass spectrum of digests of BSA obtained by using the CS/HA multilayer-modified microchip reactor with a flow rate of $2.0 \mu \mathrm{Lmin}^{-1}$. Protein concentrations are $0.20 \mu \mathrm{g} \mu \mathrm{L}^{-1}$ in $\mathrm{NH}_{4} \mathrm{HCO}_{3}$ buffer solution $\left(10 \mathrm{mmolL}^{-1}, \mathrm{pH} 8.1\right)$. b) MALDI TOF MS spectra of the digests of BSA obtained by using in-solution proteolysis with digestion time $12 \mathrm{~h}$ (trypsin/substrate ratio, 1:40). Eluent/matrix ratio, 1:1.

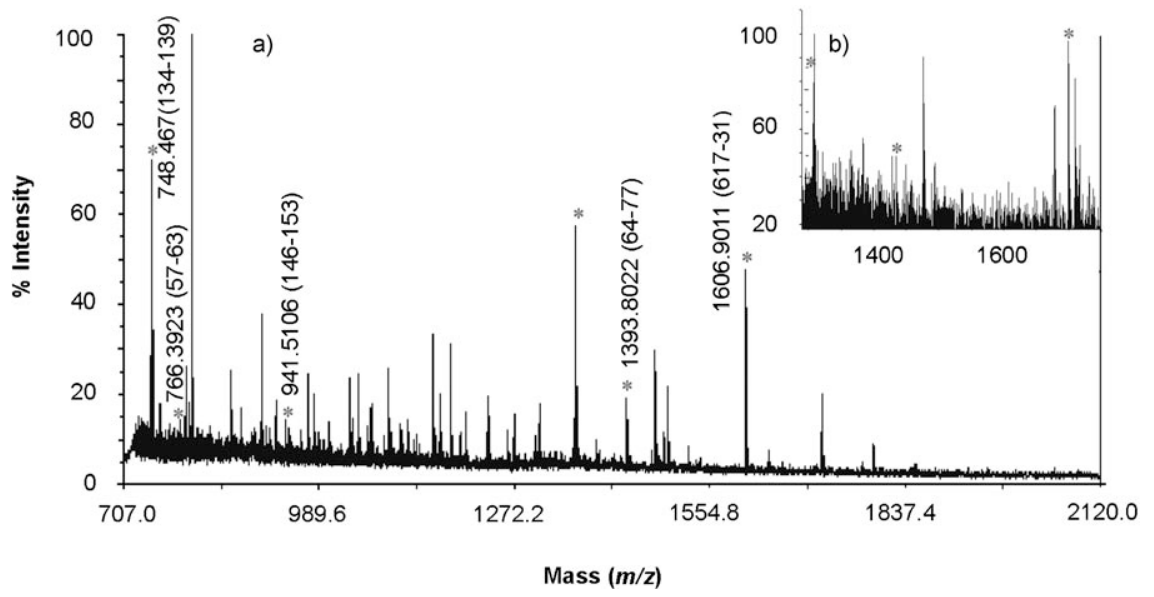

Figure 5. MALDI-TOF mass spectrum of digests of (a) $1 \mathrm{ng} \mu \mathrm{L}^{-1}$ myoglobin, and (b) $0.1 \mathrm{ng} \mu \mathrm{L}^{-1} \mathrm{Cyt}-\mathrm{c}$ in $\mathrm{NH}_{4} \mathrm{HCO}_{3}$ buffer solution $\left(10 \mathrm{mmolL}^{-1}, \mathrm{pH} 8.1\right)$ obtained by using the CS/HA-modified microchip reactor with a flow rate of $2.0 \mu \mathrm{L} \mathrm{min}^{-1}$. Eluent/matrix ratio, $1: 1$

autolysis in the microchip reactor is favorable for the positive identification of low-abundancy proteins. Additionally, Cyt-c at a low level of $0.1 \mathrm{ng} \mu \mathrm{L}^{-1}$ was digested by using the reactor, as shown in Figure 5b. Three tryptic peptides from Cyt-c are assigned. The detection limit can reach a few femtomoles for proteins and the sample size is as low as $0.05 \mathrm{ng}$ per analysis. This property minimizes the sample size, compared with the common tryptic reactions.

Furthermore, the advantage of this method is the identification of complex proteins. For example, casein extracted from bovine milk consists of four principal primary subtypes, $\alpha \mathrm{S} 1-, \alpha \mathrm{S} 2-, \beta-$, and $\kappa$-casein. The four main proteins differ not only in amino-acid sequence and length, but also vary in the degree of phosphorylation and glycosylation, disulfide-linked polymerization, and genetic polymorphism. ${ }^{[18]}$ Casein was tryptically digested by using this on-chip micro- reactor. The resulting mass spectrum is shown in Figure 6. $\alpha \mathrm{S} 1$-casein (seven peptides), $\alpha \mathrm{S} 2$-casein (seven peptides), and $\beta$-casein (four peptides) were identified by using GPS Explore Software with the integrated Mascot search-engine software. In contrast, peptides of glycosylated $\kappa$-casein were not observed by PMF. As for other available approaches, characterization of post-translational proteins is limited and additional techniques are required.

The desirable objective is to extend this microchip system for the analysis of practical biological samples. Hepatitis A virus (HAV) is exemplified to evaluate the performance of the proposed reactor. HAV causes acute hepatitis A, a serious human disease that accounts for $50 \%$ epidemic hepatitis every year. Although research of HAV has made great progress, the nosogenesis of HAV has not yet been elucidated. It is imperative to find a rapid and effective method to identify the proteins in HAV to accelerate the development of detection technology. HAV, a single-strand RNA virus, is a spherical particle of diameter $27-32 \mathrm{~nm}$ and is a symmetrical icosahedron. The virus capsid of HAV is composed of 60 protein subunits and every subunit contains four kinds of peptides. In consideration of safety, an attenuated hepatitis A vaccine was used in this research. To avoid being affected by its surrounding environment, the HAV vaccine contains several chemical and biological additives that usually deactivate the free trypsin. In this study, the vaccine was digested by using this microchip reactor and the resulting peptides were identified by using MALDI-TOF MS and the Mascot search-engine software of virus database. The results are summarized in Table 1 . Six possible proteins were detected. This is a general and effective on-chip protolysis approach for identifying proteins, and can be applied in rapid and high-throughput biological and clinical analyses. 


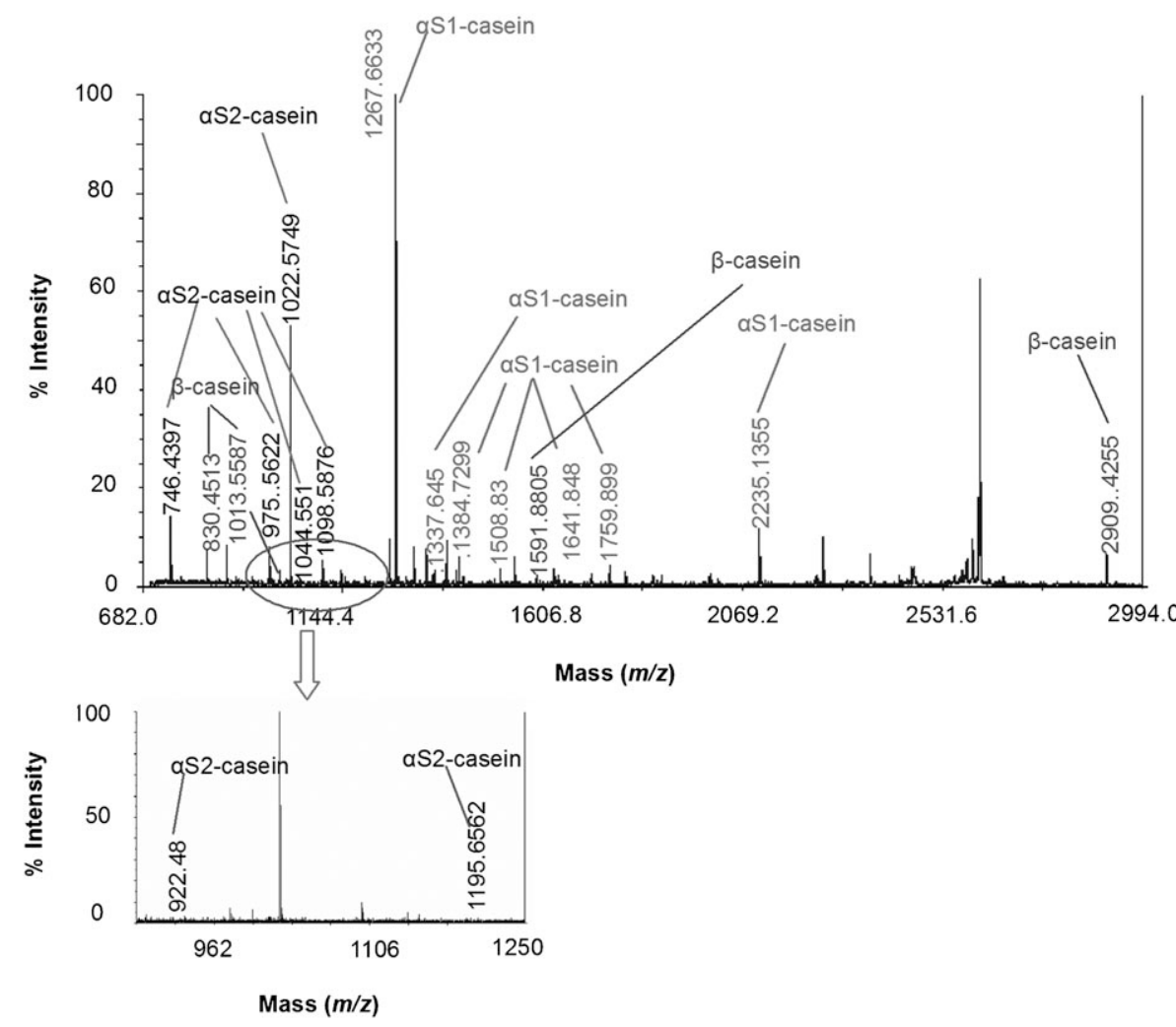

Figure 6. MALDI-TOF mass spectrum of digests of casein obtained by using the CS/HA-modified microchip reactor. Protein concentrations are $0.20 \mu \mathrm{g} \mu \mathrm{L}^{-1}$ in $\mathrm{NH}_{4} \mathrm{HCO}_{3}$ buffer solution $\left(10 \mathrm{mmol} \mathrm{L}{ }^{-1}\right.$, $\mathrm{pH}$ 8.1). Eluent/ matrix ratio, $1: 1$

Table 1. Identification of proteins of the HAV vaccine obtained from digestion by using the CS/HA-assembled microchip reactor.

\begin{tabular}{lcl}
\hline Protein & Molecular weight & Peptide match \\
\hline nucleocapsid protein & 58096 & 10 \\
nonstructural polyprotein & 185108 & 16 \\
capsid protein VP1 & 81663 & 15 \\
RNA-directed RNA polymerase & & \\
subunit VP1 & 124923 & 24 \\
outer capsid protein VP2 & 123982 & 12 \\
genome polyprotein & 367338 & 40 \\
\hline
\end{tabular}

\section{Conclusion}

We have constructed a stable biocompatible interface for trypsin immobilization on the basis of CS/HA multilayer assembly to yield high enzyme loading with well-preserved bioactivity. Digestion of proteins was performed within the proposed on-chip microreactor prior to identification by MALDI-TOF MS. The remarkable enhancement of proteolytic efficiency is attributed to the biocompatible surface and microstructure confinement. The advantage of this method lies in its flexibility, which implies that the approach can be applied in automated high-throughput analysis by using a parallel channel microchip platform integrated with the liquid chromatography or two-dimensional-gel separation procedures for original complex protein mapping. Be- sides chitosan and hyaluronic acid, other natural polyelectrolytes, such as collagen, alginate, and different glycosaminoglycans, may enhance biocompatible multilayer architecture for the functionalization of substrates, so that various biomolecules of interest can be encapsulated and stabilized in the designed scaffold. It can be anticipated that the layer-bylayer assembly is a facile and versatile approach for microchip modification and biomolecule stationary-phase patterning, which would expand potential applications in the fields of chemical biology and biotechnology.

\section{Experimental Section}

The microchip was fabricated by UV laser photoablation and thermal lamination as previously reported. ${ }^{[19]}$ Briefly, the PET sheet (100 $\mu \mathrm{m}$ thick) was placed on computer-controlled $X Y$ translation stages and scanned to be photoablated by a UV excimer laser (ArF $193 \mathrm{~nm}$ ) for generating a 2-cm long channel with a trapezoidal cross-section shape of depth $42 \mu \mathrm{m}$ and width $100 \mu \mathrm{m}$. The straight-line channel was terminated on both sides by photoablated reservoirs. The PET microchannel was thermally sealed with a PET film by using a lamination machine (Morane, UK)

Firstly, the PET microchannel was hydrolyzed with $\mathrm{NaOH}(1 \mathrm{M})$ for $20 \mathrm{~min}$ at $60^{\circ} \mathrm{C}$ followed by rinsing with $\mathrm{HCl}(0.1 \mathrm{M})$, water, and ethanol, and parts of the surface ester groups were converted to free $\mathrm{OH}$ or $\mathrm{COO}$ groups. Next, positively charged chitosan and negatively charged hyaluronic acid were deposited successively onto the hydrolyzed PET surface by incubating the latter in aqueous solutions of chitosan $(1.0 \mathrm{wt} \%$, medical grade, Qingdao Heppe Biotechnology Co.) and hyaluronic acid (1.0 mg mL ${ }^{-1}, \mathrm{CPN}$, Czech Republic), respectively, each for $30 \mathrm{~min}$. This procedure was repeated five times until a stable film was obtained. After the five layers $\left(\mathrm{PET}_{0} / \mathrm{CS} 1 / \mathrm{HA} 2 / \mathrm{CS} 3 / \mathrm{HA} 4 / \mathrm{CS} 5\right)$ had been built, an further enzyme-loading step was performed. On top of this stable precursor system, several multilayers of HA/CS-trypsin were deposited by immersing the microchip alternately in trypsin solution $\left(5 \mathrm{mg} \mathrm{mL}^{-1}\right.$, in tris- $\mathrm{HCl}$ buffer (50 mm) containing $\mathrm{CaCl}_{2}(20 \mathrm{~mm}), \mathrm{pH}$ 8.0, Sigma Company, USA) for $3 \mathrm{~h}$ at $4{ }^{\circ} \mathrm{C}$, and aqueous solutions of hyaluronic acid and chitosan, respectively, followed by sufficient washing with water. On top of the HA/ CS-trypsin layers, a final layer of trypsin was adsorbed. This multilayer system was fabricated on the PET microchip as a bioreactor.

The surface topography was assessed by using an atomic force microscope (EC-SPM, Picoscan 2100) in tapping mode. Micron scanning was performed with nanosensors etched silicon probes. The instrument parameters were set as follows: scan size $3.5 \mu \mathrm{m}$, resonance frequency 60 $80 \mathrm{KHz}$. The images were flattened by using the Nanoscope software.

The controlled immobilization of trypsin in well-interconnected macroporous membranes was performed in situ by using a quartz-crystal microbalance analyzer (CHI420, CH Instruments, USA) and quartz crystals (7.995 MHz) sandwiched between two Au electrodes. Chitosan, HA, and 
trypsin solution were assembled onto the gold surfaces. After deposition, the gold electrode surface was rinsed thoroughly with pure water and dried under nitrogen gas. The QCM frequency change in air was measured.

The activity of the encapsulated enzyme in the microchip was examined by pumping a solution of $\alpha-N$-benzoyl-L-arginine ethyl ester (BAEE) in tris-HCL $(50 \mathrm{~mm})$ buffer $(\mathrm{pH} 8.0)$ through the trypsin-adsorbing PET channel at a flow rate of $100 \mu \mathrm{Lh}^{-1}$ by using a syringe pump. The resulting products were analyzed by using a capillary electrophoresis system (P/ACE System 5000, Beckman). The capillary electrophoresis was run in phosphate buffer solution $(\mathrm{pH} 2.5)$ at $15^{\circ} \mathrm{C}$ with UV detection at $214 \mathrm{~nm}$. The trypsin activity was calculated from the flow rate and absorbance difference.

Protein solutions of BSA (Bio Basic, Toronto, Canada), cytochrome c, myoglobin, casein extracted from bovine milk (Sigma Company, USA), and hepatitis A vaccine (Changsheng Industry Co., Changchun, China) in $\mathrm{NH}_{4} \mathrm{HCO}_{3}(10 \mathrm{~mm})$ buffer solution $(\mathrm{pH}$ 8.1) were driven through the LBL-modified PET microchannel by using a syringe pump at a flow rate of $120 \mu \mathrm{Lh}^{-1}$. Effluents accumulated in the reservoir were collected and then identified by MALDI-TOF MS. All MS experiments were performed by using a 4700 Proteomics Analyzer with TOF/TOF optics. Each volume of sample solution $(0.5 \mu \mathrm{L})$ was dropped onto the MALDI plate. After the solvent was evaporated, a volume of matrix solution $(0.25 \mu \mathrm{L}$, $4 \mathrm{mg} \mathrm{mL}^{-1} \alpha$-cyano-4-hydroxycinnamic acid in acetonitrile $(50 \%)$ with TFA $(0.1 \%))$ was dropped onto the dried samples. Before identification of the samples, the MS instrument was calibrated by using an internal calibration mode with tryptic peptides of myoglobin. All spectra were recorded from signal averaging of 800 laser shots with the laser intensity kept constant. GPS Explorer software from Applied Biosystems with Mascot as a search engine and SwissProt (version of 050303) as a database was used to identify proteins. All proteins were identified by using the peptide fingerprint mass spectra combined with tandem mass spectra. The peptide mass tolerance was set to $80 \mathrm{ppm}$, and the tandem mass tolerance was set to $0.5 \mathrm{Da}$.

\section{Acknowledgements}

This work was supported by the National Natural Science Foundation of China (20575013), the National Basic Research Priorities Program 2001CB510202, STCSM 05QMH1402, and EPFL through a visiting fellowship to B.H.L. The authors thank Dr. A. Lionello (EPFL) for helpful discussion and V. Devaud (EPFL) for technical help.

[1] a) J. Lahann, M. Balcells, H. Lu, T. Rodon, K. F. Jensen, R. Langer, Anal. Chem. 2003, 75, 2117-2122; b) K. S. Philips, Q. Cheng, Anal. Chem. 2005, 77, 327-334.

[2] a) F. Xu, G. L. Zhen, F. Yu, E. Kuennemann, M. Textor, W. Knoll, $J$. Am. Chem. Soc. 2005, 127, 13084-13085; b) Y. Lvov, F. Caruso, Anal. Chem. 2001, 73, 4212-4217.
[3] a) D. Niwa, Y. Yamada, T. Homma, T. Osaka, J. Phys. Chem. B 2004, 108, 3240-3245; b) M. Curreli, C. Li, Y. Sun, B. Lei, M. A. Gundersen, M. E. Thompson, C. W. Zhou, J. Am. Chem. Soc. 2005, 127, 6922-6923.

[4] A. Langner, A. Panarello, S. Rivillon, O. Vassylyev, J. G. Khinast, Y. J. Chabal , J. Am. Chem. Soc. 2005, 127, 12798-12799.

[5] a) Z. Y. Park, D. H. Russell, Anal. Chem. 2000, 72, 2667-2670; b) J. Fan, W. Q. Shui, P. Y. Yang, X. Y. Wang, Y. M. Xu, H. H. Wang, X. Chen, D. Y. Zhao, Chem. Eur. J. 2005, 11, 5391-5396.

[6] a) D. S. Peterson, T. Rohr, F. Svec, J. M. J. Fréchet, Anal. Chem 2002, 74, 4081-4088; b) K. Sakai-Kato, M. Kato, T. Toyo'oka, Anal. Chem. 2002, 74, 2943-2949.

[7] a) H. Y. Qu, H. T. Wang, Y. Huang, W. Zhong, H. J. Lu, J. L. Kong, P. Y. Yang, B. H. Liu, Anal. Chem. 2004, 76, 6426-6433; b) H. L. Wu, Y. P. Tian, B. H. Liu, H. J. Lu, X. Y. Wang, J. J. Zhai, H. Jin, P. Y. Yang, Y. M. Xu, H. H. Wang, J. Proteome Res. 2004, 3, $1201-$ 1209; c) Y. Liu, H. J. Lu, W. Zhong, P. Y. Song, J. L. Kong, P. Y. Yang, H. H. Girault, B. H. Liu, Anal. Chem. 2006, 78, 801-808.

[8] a) G. Decher, Science 1997, 277, 1232-1237; b) T. Groth, A. Lendlein, Angew. Chem. 2004, 116, 944-946; Angew. Chem. Int. Ed. 2004, 43, 926-928.

[9] a) D. S. Salloum, J. B. Schlenoff, Biomacromolecules 2004, 5, 10891096; b) R. J. Pei, X. Q. Cui, X. R. Yang, E. K. Wang, Biomacromolecules 2001, 2, 463-468; c) I. Ichinose, R. Takaki, K. Kuroiwa, T. Kunitake, Langmuir 2003, 19, 3883-3888.

[10] a) B. Thierry, F. M. Winnik, Y. Merhi, M. Tabrizian, J. Am. Chem. Soc. 2003, 125, 7494-7495; b) B. Thierry, P. Kujawa, C. Tkaczyk, F. M. Winnik, L. Bilodeau, M. Tabrizian, J. Am. Chem. Soc. 2005, $127,1626-1627$

[11] T. C. Rohner, J. Josserand, H. Jensen, H. H. Girault, Anal. Chem. 2003, 75, 2065-2074.

[12] C. H. Lei, S. Shin, J. Liu, E. J. Ackerman, J. Am. Chem. Soc. 2002 , $124,11242-11243$.

[13] G. T. Saccone, J. D. Skinner, L. A. Burgoyne, FEBS Lett. 1983, 157, 111-114.

[14] a) W. Jin, X. Y. Shi, F. Caruso, J. Am. Chem. Soc. 2001, 123, 81218122; b) F. Caruso, C. Schüler, Langmuir 2000, 16, 9595-9603.

[15] a) A. Lionello, J. Josserand, H. Jensen, H. H. Girault, Lab chip 2005, 5, 254-260; b) A. Lionello, J. Josserand, H. Jensen, H. H. Girault, Lab chip 2005, 5, 1096-1103.

[16] A. N. Zelenetskii, T. A. Akopova, N. R. Kildeeva, G. A. Vikhoreava, E. S. Obolonkova, A. A. Zharov, Russ. Chem. Bull. 2003, 52, $2073-$ 2077.

[17] a) D. Craft, A. Doucette, L. Li, J. Proteome Res. 2002, 1, 537-547; b) A. Doucette, D. Craft, L. Li, Anal. Chem. 2000, 72, 3355-3362.

[18] a) A. M. Fiat, P. Jolles, Mol. Cell. Biochem. 1989, 87, 5-30; b) S. G. Rival, S. Fornaroli, C. G. Boeriu, H. J. Wichers, J. Agric. Food Chem. 2001, 49, 287-294.

[19] M. A. Roberts, J. S. Rossier, P. Bercier, H. H. Girault, Anal. Chem. 1997, 69, 2035-2042.

Received: December 23, 2005 Revised: February 20, 2006 Published online: June 26, 2006 